\title{
Endocannabinoid and ceramide levels are altered in patients with colorectal cancer
}

\author{
LING CHEN, HUIXIA CHEN, YANTING LI, LEI LI, YAN QIU and JIE REN
}

Department of Medical Sciences, Medical College, Xiamen University, Xiamen 361102, P.R. China

Received March 9, 2015; Accepted April 27, 2015

DOI: $10.3892 /$ or.2015.3973

\begin{abstract}
Endocannabinoids and ceramides have demonstrated growth inhibition, cell death induction and pro-apoptotic activity in cancer research. In the present study, we describe the profiles of two major endocannabinoids, ceramides, free fatty acids and relevant metabolic enzymes in 47 pairs of human colorectal cancer tissues and adjacent non-tumor tissues. Among them, anandamide (AEA) and its metabolite, arachidonic acid (AA), were markedly upregulated in cancer tissues particularly in those with lymphatic metastasis. The levels of C16 and C24 ceramides were significantly elevated in the colorectal tumor tissues, while levels of C18 and C20 ceramides showed opposite trends. Levels of two enzymes participating in the biosynthesis and degradation of AEA, $N$-acyl-phosphatidylethanolamine-hydrolyzing phospholipase D (NPLD) and fatty acid amide hydrolase (FAAH), together with the most abundant ceramide synthases (CerS1, CerS2, CerS5 and CerS6) in the colon were also determined. Quantitative-PCR analysis indicated that the mRNA levels of these enzymes were overexpressed in the tumor tissues. The activities of NPLD and FAAH were also upregulated. In addition, both gene and protein expression levels of cannabinoid receptor 1 (CB1) were elevated but not of $\mathrm{CB} 2$. Elevation of AEA and alteration of ceramides (C16, C24, C18, C20) may qualify as potential endogenous biomarkers and novel drug targets for colorectal cancer.
\end{abstract}

\section{Introduction}

As one of the three most common cancers in the world, colorectal cancer (CRC) occurs with a global incidence exceeding 1.2 million new cases and 600,000 deaths per year. Endoscopic removal of precursor lesions appears to be the only effective treatment strategy for CRC (1). The most frequently used chemotherapy regimens for CRC to date are respective combinations of leucovorin, oxaliplatin, irinotecan

Correspondence to: Dr Jie Ren, Department of Medical Sciences, Medical College, Xiamen University, Xiamen 361102, P.R. China E-mail: renjie_7912@xmu.edu.cn

Key words: endocannabinoids, anandamide, ceramides, colorectal cancer and fluorouracil (5-FU), which are not highly efficient target therapies, and are accompanied by a series of adverse side effects, such as peripheral nerve toxicity and severe neutrophil reduction $(2,3)$. It is essential to identify and verify potential endogenic biomarkers for the diagnosis, therapy and further prevention of CRC. Early-stage CRC is usually misdiagnosed as chronic appendicitis or cholelithiasis. The carcinoembryonic antigen (CEA) level in patient blood is a common chemical marker for the clinical diagnosis of CRC, yet a high CEA level often suggests that tumors have spread to distant sites. Therefore, it is necessary to explore potential therapeutic biomarkers and targets which may be effective with fewer side effects. Here, we suggest that endogenetic cannabinoids and ceramides may be a possible option.

As two major endocannabinoids, anandamide (AEA) was first isolated, followed by $2-\mathrm{AG}(4,5)$, and both are found within the gastrointestinal tract (6). $N$-acyl-phosphatidylethanolaminehydrolyzing phospholipase D (NPLD) is a key enzyme that participates in a series of AEA synthesis reactions, and synthesizes and releases AEA from the direct biosynthetic precursor $N$-arachidonoylphosphatidylethanolamine (NAPE) $(7,8)$. Fatty acid amide hydrolase (FAAH) acts as the main hydrolytic enzyme for AEA, which is widely distributed in tissues containing the CB1 and CB2 receptors (9), and mainly catalyzes AEA into arachidonic acid (AA) and ethanolamine. Diacylglycerol lipase- $\alpha$ (DGL- $\alpha$ ) and monoacylglycerol lipase (MGL) are respectively responsible for 2-AG biosynthesis and hydrolysis (8). Both AEA and 2-AG have been verified to bind to $\mathrm{CB}$ receptors. The $\mathrm{CB} 1$ receptor is not only distributed in regions of the brain for central nerve system control, but is also present in the prostate, spleen, lymphocytes and small intestine $(10,11)$. The CB2 receptor is mainly found in immune tissues and immunocompetent blood cells, and is highly expressed in natural killer cells, mast cells and monocytes (11). AEA seems to have more affinity for the $\mathrm{CB} 1$ receptor than $\mathrm{CB} 2$, but 2-AG prefers to bind to the CB2 receptor $(12,13)$. Recently, a series of studies investigated the antineoplastic role of endocannabinoids (14). AEA efficaciously inhibited the proliferation of multiple tumor cell lines with $\mathrm{CB}$ receptormediated antitumor effects, including human breast, prostatic and colorectal cancer cell lines (15-17). De Petrocellis et al reported that AEA prevented the proliferation of cancer cells by inhibiting DNA synthesis and arresting the G1/S transition of the cell cycle, which was mainly mediated via the interaction with the transmembrane $\mathrm{CB} 1$ receptor rather than the $\mathrm{CB} 2$ 
receptor (16). Furthermore, the pro-apoptotic effect of AEA was also realized by acting on the vanilloid receptors (18). Notably, another pro-apoptotic endogenic lipid, ceramide, was also confirmed to be elevated and to participate in apoptosis via the endocannabinoid system $(16,19)$.

Ceramides are vital signaling sphingolipids involved in cell growth arrest, differentiation and apoptosis, and contain two frame structures, a sphinganine base and a fatty acyl with varying lengths of carbon chains (20). The most abundant ceramides in human tissues are those with $\mathrm{C} 16$ to C24 fatty acyl bases, which are mainly synthesized by six ceramide synthases (CerSs) (CerS1-CerS6). CerS1, CerS2, CerS5 and CerS6 are relatively plentiful in colon tissues. CerSs show different specificity to produce ceramides with differing chain lengths. CerS1 is mainly responsible for $\mathrm{C} 18$ ceramides, and CerS2 for $\mathrm{C} 20-\mathrm{C} 24$ ceramides, CerS5 for $\mathrm{C} 16$ ceramides and CerS6 for C16/18 ceramides, respectively (21). Ceramidase inhibition or application of ceramide analogues promoted the apoptosis of human CRC cells (22). However, pharmacological inhibition of ceramide biosynthesis protected cancer cells from apoptosis (23). In addition, cancer cell lines with low ceramide levels or a defect in ceramide synthesis can escape from apoptosis induced by radiation (24). One study found that patients with head and neck cancer resistant to radiotherapy had decreased ceramide levels in tumor tissues (25). Higher or lower levels of ceramide appear to be closely linked to cancer cell apoptosis or malignant progression. Hence, in addition to endocannabinoids, ceramides may be another group of important lipids involved in CRC progression.

In order to reveal the expression patterns of endocannabinoids and ceramides in human CRC, we evaluated the endogenic levels of AEA, 2-AG, ceramides and their metabolites via liquid chromatography-tandem mass spectrometry (LC-MS/MS). The expression levels of two cannabinoid receptors and the relevant enzymes, such as NPLD, FAAH, DGL- $\alpha$, MGL and CerSs, were also analyzed to evaluate the potential effects of these lipids on tumor treatment.

\section{Materials and methods}

Patients and tissue sampling. Colorectal cancer samples were obtained from 47 Chinese patients undergoing surgical resection at the Department of Colorectal Surgery, Fujian Medical University Union Hospital (Fuzhou, China) during 2011, and were confirmed with TNM stage according to the World Health Organization TNM staging 7th edition by pathological analysis. Non-tumor control tissues were resected from the same patients at the region 5-7 $\mathrm{cm}$ above the tumor position. All tissues were divided into several slices for lipid, gene and protein analysis, and were freshly frozen and stored at $-80^{\circ} \mathrm{C}$ immediately after resection. Informed consent was obtained from each patient before surgery. Study of the resected human tissues was approved by the Ethics Committee of Fujian Medical University Union Hospital, following clinical registration guidelines in China.

Lipid extraction and analysis. Lipids were extracted from the tissue samples using a previously described method with modifications $(26,27)$. In brief, the samples were homogenized in methanol/ $\mathrm{H}_{2} \mathrm{O}(\mathrm{v} / \mathrm{v}, 50 / 50)$ containing internal standards
(C17:1 FAE, C17:0 heptadecanoic acid and C17:0 ceramide) and were then extracted with chloroform. The organic phase was transferred and dried under nitrogen flow $\left(\mathrm{N}_{2}\right)$. After being redissolved with chloroform, the lipid solution underwent solid-phase extraction, and was eluted by methanol/ chloroform $(\mathrm{v} / \mathrm{v}, 10 / 90)$. The elution containing endocannabinoids and ceramides was dried and reconstituted with $100 \mu \mathrm{l}$ of methanol/chloroform ( $\mathrm{v} / \mathrm{v}, 75 / 25)$, and then $10 \mu \mathrm{l}$ of this elution was used for detection using the $3200 \mathrm{Q}$ Trap LC-MS/MS system (Applied Biosystems, USA) coupling with the 1100-LC system (Agilent, China). The mobile phase for endocannabinoids and ceramides consisted of methanol and ultra-pure water ( $\mathrm{pH} 7.4)$, and the gradient elution was as follows: 85\% methanol was kept for the first $3 \mathrm{~min}$, followed by a linear gradient from 85 to $100 \%$ methanol for $2 \mathrm{~min}$ and then $100 \%$ methanol was continued for $15 \mathrm{~min}$. The elution condition was finally returned to $85 \%$ methanol at a flow rate of $0.7 \mathrm{ml} / \mathrm{min}$. The column temperature was maintained at $40^{\circ} \mathrm{C}$. Ion detection was monitored by $\mathrm{APCI}{ }^{+}-\mathrm{MRM}$ mode. The molecular ions were monitored at $\mathrm{m} / \mathrm{z} 348.00 / 62.00$ for AEA, $m / z$ 379.10/287.10 for 2-AG, $m / z, 313.1 / 62.0$ for C17:1 FAE, $m / z$ 520.4/264.2 for C16:0 ceramide, $\mathrm{m} / \mathrm{z}$ 534.3/264.2 for C17:0 ceramide, $\mathrm{m} / \mathrm{z}$ 548.4/264.2 for C18:0 ceramide, $\mathrm{m} / \mathrm{z}$ 576.4/264.2 for C20:0 ceramide, $\mathrm{m} / \mathrm{z}$ 632.4/264.2 for C24:0 ceramide and $\mathrm{m} / \mathrm{z}$ 630.4/264.2 for C24:1 ceramide. Elution condition, ion monitor model and the molecular ion parameters of free fatty acids were previously described in detail (26).

Assessment of enzyme activity. Analysis of enzyme activity was also carried out using a previously described method (27). Samples were homogenized in $50 \mathrm{mM}$ Tris- $\mathrm{HCl}$ buffer (pH 7.4) containing $0.32 \mathrm{M}$ sucrose to obtain the total proteins. For NPLD activity, in $50 \mathrm{mM}$ Tris-HCl buffer $(0.1 \%$ Triton $\mathrm{X}-100$ and $1 \mathrm{mM}$ phenylsulphonylfluoride), $100 \mu \mathrm{g}$ of sample protein was incubated with $50 \mu \mathrm{M}$ 1-palmitoyl,2-palmitoylsn-glycero-3-phosphoethanolamine-N-heptadecenoyl (C17:1 NAPE) at $37^{\circ} \mathrm{C}$ for $30 \mathrm{~min}$, and then $0.2 \mathrm{ml}$ methanol containing $\left[{ }^{2} \mathrm{H}_{4}\right]$-OEA was added to halt the reaction. DGL- $\alpha$ activity was measured at $37^{\circ} \mathrm{C}$ for $30 \mathrm{~min}$ in $50 \mathrm{mM}$ Tris- $\mathrm{HCl}$ containing $0.1 \%$ Triton $\mathrm{X}-100$. The reaction between the sample proteins and $50 \mu \mathrm{M}$ diheptadecanoyl-sn-glycerol as substrates were stopped by $0.2 \mathrm{ml}$ methanol containing C17:1 FAE. FAAH and MGL activity were measured by incubating the sample proteins with AEA or 2-oleoylglycerol as respective substrates in Tris- $\mathrm{HCl}$ buffer containing fatty acid-free BSA $(0.05 \%)$. The reactions were also terminated by adding $0.2 \mathrm{ml}$ methanol containing C17:0 heptadecanoic acid. The reaction products of the enzyme assays above were monitored by LC-MS and the molecular ions were: $\mathrm{m} / \mathrm{z} 312.1 / 62.0$ for heptadecanoylethanolamide, $m / z, 330.1 / 66.0$ for $\left[{ }^{2} \mathrm{H}_{4}\right]$-OEA, $m / z, 303$ for arachidonic acid, $m / z, 281$ for oleic acid, $m / z, 269$ for 17:0 heptadecanoic acid, and $m / z 367.1$ for 1(3)-heptadecanoyl-sn-glycerol (HDG).

Evaluation of protein and $m R N A$ levels. Protein expression levels of the tissue samples were evaluated by western blot analysis. First, the concentration of homogenized tissue protein was measured using the BCA protein assay kit (Pierce, China). Proteins were separated by sodium dodecyl sulfate polyacrylamide gel electrophoresis (SDS-PAGE), and then transferred to PVDF membranes (Amersham Biosciences, 
Table I. Characteristics of the CRC patients.

\begin{tabular}{lrr}
\hline Characteristics & \multicolumn{2}{c}{ Data } \\
\hline Age (years) & \multicolumn{2}{c}{60} \\
Median & \multicolumn{2}{c}{$33-88$} \\
Range & & \\
Gender, n (\%) & 31 & $(66)$ \\
Male & 16 & $(34)$ \\
Female & & \\
Tumor stage, n (\%) & 2 & $(4)$ \\
T2 & 32 & $(68)$ \\
T3 & 13 & $(28)$ \\
T4 & & \\
Nodal status, $\mathrm{n}(\%)$ & 22 & $(47)$ \\
N0 & 25 & $(53)$ \\
N1+ & & \\
TNM staging, n (\%) & 1 & $(2)$ \\
I & 21 & $(45)$ \\
II & 20 & $(42)$ \\
III & 5 & $(11)$ \\
IV & & \\
& &
\end{tabular}

China). Membranes with the proteins were incubated overnight with primary antibodies against the $\mathrm{CB} 1$ receptor (1:500), CB2 receptor (1:500; Cayman, China) or $\beta$-actin at $4^{\circ} \mathrm{C}$, followed by incubation with HRP-conjugated anti-rabbit $\mathrm{IgG}$ antibody (1:10,000; Sigma, China) for $1 \mathrm{~h}$ at room temperature. Protein bands were visualized using an ECL Plus kit (Amersham Biosciences). mRNA expression levels were measured by RT-qPCR assay. Tissue samples were ground using ice-cold porcelain mortars with TRIzol to extract total RNA. The RNA concentration was measured with a spectrophotometer (Beckman Coulter, USA). Reverse transcription was completed with a ReverTra Ace qPCR RT kit (Toyobo, Japan) following the manufacturer's instructions. Finally quantitative PCR was performed and analyzed in a 7500 Fast real-time PCR System (Applied Biosystems). The mRNA expression levels were normalized by housekeeping gene $18 \mathrm{~S}$ as the internal standard.

Primers were as follows: NPLD forward primer (F), 5'-TG GCTGGGACACGCG-3' and reverse primer (R), 5'-GGG ATCCGTGAGGAGGATG-3'; FAAH F, 5'-GCCTCAAGG AATGCTTCAGC-3' and R, 5'-TGCCCTCATTCAGGCTCA AG-3'; MGL F, 5'-CATGTGGATTCCATGCAGAAAG-3' and R, 5'-AGGATTGGCAAGAACCAGAGG-3'; DGL- $\alpha$ F, 5'-AGAATGTCACCCTCGGAATGG-3' and R, 5'-GTGGCT CTCAGCTTGACAAAGG-3'; CB1 F, 5'-AGCCTCTGGATA ACAGCATGG-3' and R, 5'-AATCTTGACCGTGCTCTT GATG-3'; CB2 F, 5'-CTCAGTGACCAGGTCAAGAAGG-3' and R, 5'-TTTTGCCTCTGACCCAAGG-3'; CerS1 F, 5'-TTT GGCTCCCGCACAATGT-3' and R, 5'-AAAAGCGAGATA GAGGTCCTCA-3'; CerS2 F, 5'-GCTCTTCCTCATCGTTCG ATAC-3' and R, 5'-GTGTAGCCACGTACAGCTCA-3'; CerS5 F, 5'-GCTGCTCTTCGAGCGATTTAT-3' and R, 5'-CCT CCGATGGCGAAACCAG-3'; CerS6 F, 5'-TTTGGCTCC CGCACAATGT-3' and R, 5'-AAAAGCGAGATAGAGGTC
CTCA-3'; 18S F, 5'-CAGCCACCCGAGATTGAGCA-3' and R, 5'-TAGTAGCGACGGGCGGTGTG-3'.

Statistical analysis. Data of the results are expressed as means \pm SEM. Statistical significance was analyzed with one-way variance analysis (ANOVA) followed by the Bonferroni all pairs test. Statistical analysis was completed using GraphPad Prism (GraphPad Software, USA), and the difference was considered statistically significant at $\mathrm{p}<0.05$.

\section{Results}

Characteristics of the patients. Tumor samples were collected from 47 patients with CRC during surgical removal. Detailed baseline characteristics of the 47 patients are listed in Table I. Their median age was 60 years (range, 33 to 88). Sixty four percent of the patients were male. Approximately $68 \%$ of the patients were at tumor stage T3 with tumor stage T2 and T4, respectively, consisting of 4 and $28 \%$ of the cases. Regarding the nodal status, the patients were distributed almost equally between $\mathrm{N0}(47 \%)$ and N1+ (53\%). TNM staging was as follows: stage I, $2 \%$; stage II, 45\%; stage III, 42\%; stage IV, $11 \%$. Here, nodal status was applied to classify all samples because of equalization and comprehensiveness. The groups were classified as follows: N0 group, tumors with no lymph node metastasis; N1+ group, tumors with metastasis of one or more lymph nodes; and $\mathrm{N}$ group, adjacent non-tumor tissues.

Alteration of endogenic lipid levels in human CRC tissues. After detection by LC-MS/MS assay and normalization by the wet weight of each sample, the levels of AEA, 2-AG, ceramides and free fatty acids were analyzed. Regarding the endocanabinoids, the anandamide level was obviously elevated in the CRC tissues when compared with this level in the non-tumor control tissues, and was nearly 2-fold higher in the tissues with metastasis of the lymph nodes (Fig. 1A). However, change in the level of the other endocannabinoid 2-AG was not detected between the tumor tissues and the control tissues (Fig. 1B). Levels of long-chain ceramides also showed variation with different trends. Levels of C16:0 and C24:0 ceramides in human non-tumor colon tissues were, respectively, 102.6 \pm 6.0 and $8.6 \pm 0.8 \mathrm{pmol} / \mathrm{mg}$ tissue, and both were obviously increased in the tumor tissues (Fig. 2A-a and -b). A 1.5-fold higher level of $\mathrm{C} 24: 1$ ceramide was detected in the tumor tissues with lymph node metastasis (Fig. 2A-c). Elevation of C24 ceramide showed a positive correlation with lymph node invasion. In contrast, reduced levels of both $\mathrm{C} 18$ and $\mathrm{C} 20$ ceramides were observed in the tumor tissues as compared with these levels in the adjacent noncancerous tissues (Fig. 2A-d and -e). Regarding a comprehensive series of multiple free fatty acids (FFA), levels of saturated fatty acids (SFAs), monounsaturated fatty acids (MUFAs) and polyunsaturated fatty acids were also detected using the LC-MS/MS method. Unexpectedly, as the main metabolic product of AEA, levels of arachidonic acid (C20:4 FFA) were markedly increased in the tumor tissues, and were even higher in the N1+ group (Fig. 1C). In addition, compared with the non-tumor tissues, most FFAs presented upregulated trends in the CRC tissues. As for the SFAs, C18:0 and C24:0 FFAs displayed higher levels in the tumor tissues (Fig. 3). MUFAs from $\mathrm{C} 14$ to $\mathrm{C} 24$ were consistently elevated in the tumor 
A

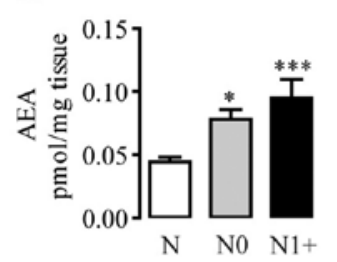

D

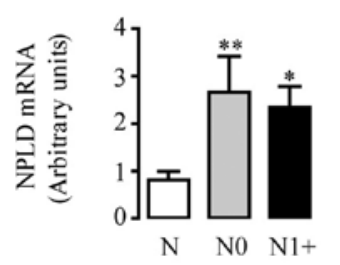

B

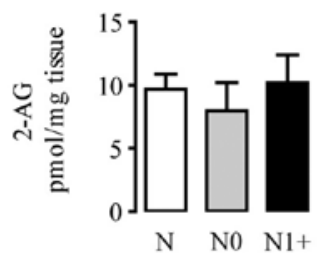

E

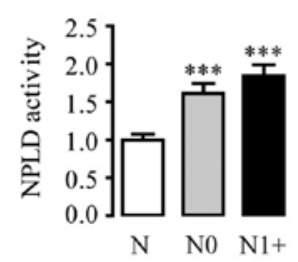

C

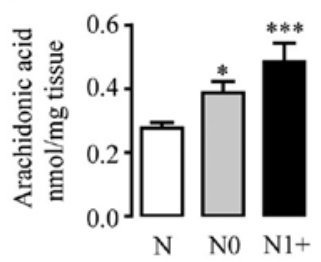

F

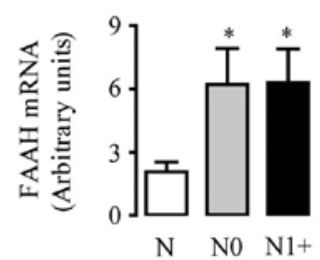

G

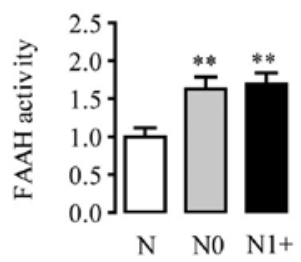

Figure 1. Alteration of endocannabinoids and corresponding metabolic enzymes in human colorectal cancer tissues. (A) Level of AEA was increased in the CRC tissues. (B) Level of 2-AG showed no difference between the tumor tissues and the control. (C) The AEA metabolite, arachidonic acid, was upregulated in the CRC tissues. (D and E) The mRNA expression and enzyme activity of NPLD were enhanced in the CRC tissues. (F and G) The mRNA expression and enzyme activity of FAAH revealed the same upward trend in the CRC tissues. Non-tumor colon tissue controls (N, open bars), CRC with no lymph node metastasis (N0, gray bar) and CRC with lymph node metastasis (N1+, solid bars). ${ }^{*} \mathrm{p}<0.05,{ }^{* * *} \mathrm{p}<0.001, \mathrm{n}=22-47$.

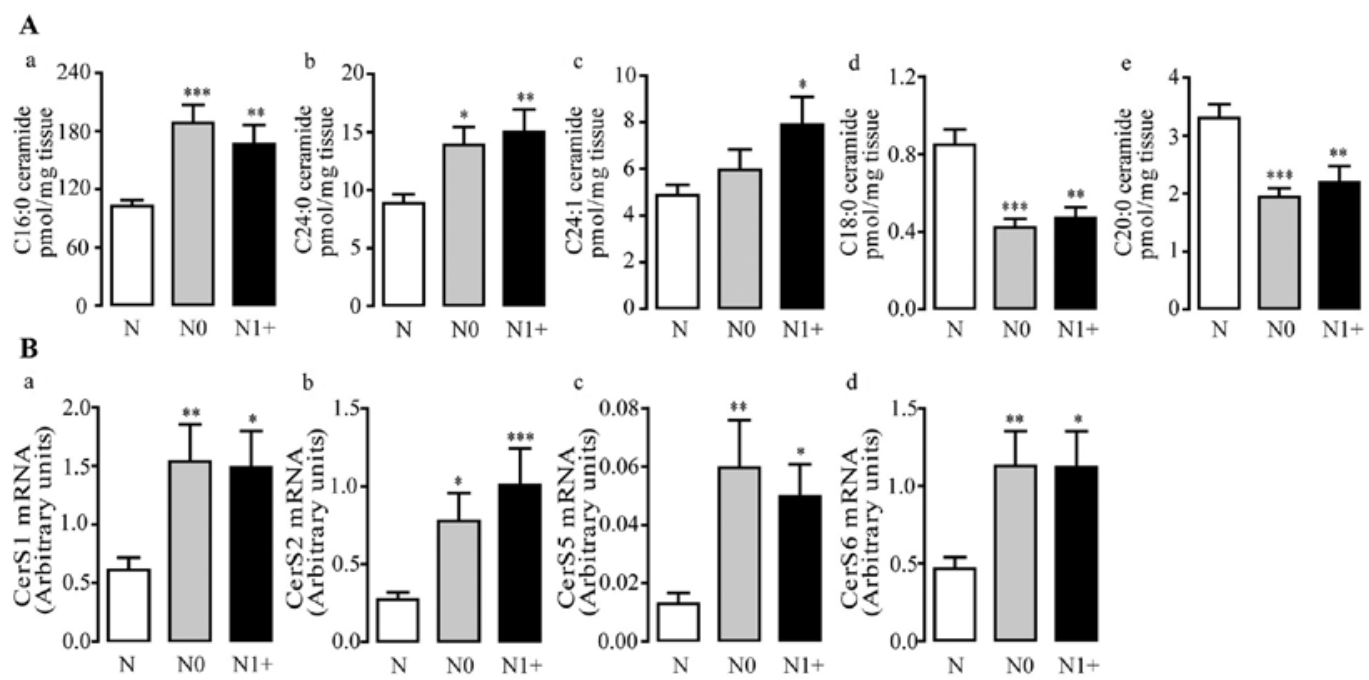

Figure 2. Varied levels of ceramides and ceramide synthases in the human colorectal cancer tissues. (A) C16:0, C24:0 and C24:1 ceramides were elevated, and C18:0/C20:0 ceramides were downregulated in the CRC tissues. (B) The mRNA levels of CerS1, CerS2, CerS5 and CerS6 were upregulated in the CRC tissues (N0, gray bar, CRC without lymph node metastasis; N1+, solid bars, CRC with lymph node metastasis) compared with the non-tumor colon tissue controls (N, open bars). ${ }^{*} \mathrm{p}<0.05,{ }^{* *} \mathrm{p}<0.01,{ }^{* * *} \mathrm{p}<0.001, \mathrm{n}=22-47$.

tissues (Fig. 4). Except C18 FFAs, most PUFAs also revealed highly increasing trends (Fig. 5).

Changes in metabolic enzymes for corresponding lipids in human CRC tissues. In order to explain the variation of lipids in human CRC, mRNA expression levels and activities of metabolic enzymes were respectively analyzed by quantitative PCR assay and enzyme activity assay as described above. We first measured the mRNA expression levels and activities of associative metabolic enzymes responsible for AEA and 2-AG. As a main synthetase of AEA, NPLD was 2- to 3-fold overexpressed in the tumor tissues; the enzyme activities of which showed the same upward trend (Fig. 1D and E). Notably, FAAH, a key hydrolase of AEA, also presented higher mRNA expression levels and catalytic activities in the CRC tissues (Fig. 1F and G). The transfer enzymes responsible for 2-AG were further detected in the same manner. The mRNA levels and catalytic activities of both synthetase DGL- $\alpha$ and hydrolase MGL exhibited no significant differences between the tumor and the control tissues. Moreover, to investigate the different variation trends of long-chain ceramides, the mRNA levels of four abundant CerSs in the human colon were also measured, that is CerS1, CerS2, CerS5 and CerS6. The results showed that all CerSs revealed 2- to 4-fold upward trends (Fig. 2B). CerS1, a synthase responsible for $\mathrm{C} 18$ ceramide synthesis, unexpectedly increased, and the same trends were also observed for CerS2 and CerS6, which partly synthesize $\mathrm{C} 20$ and $\mathrm{C} 18$ ceramides. Yet, CerS5 and CerS6 for C16 ceramide showed consistent upregulation, as did CerS2 which is partly responsible for $\mathrm{C} 24$ ceramide. 

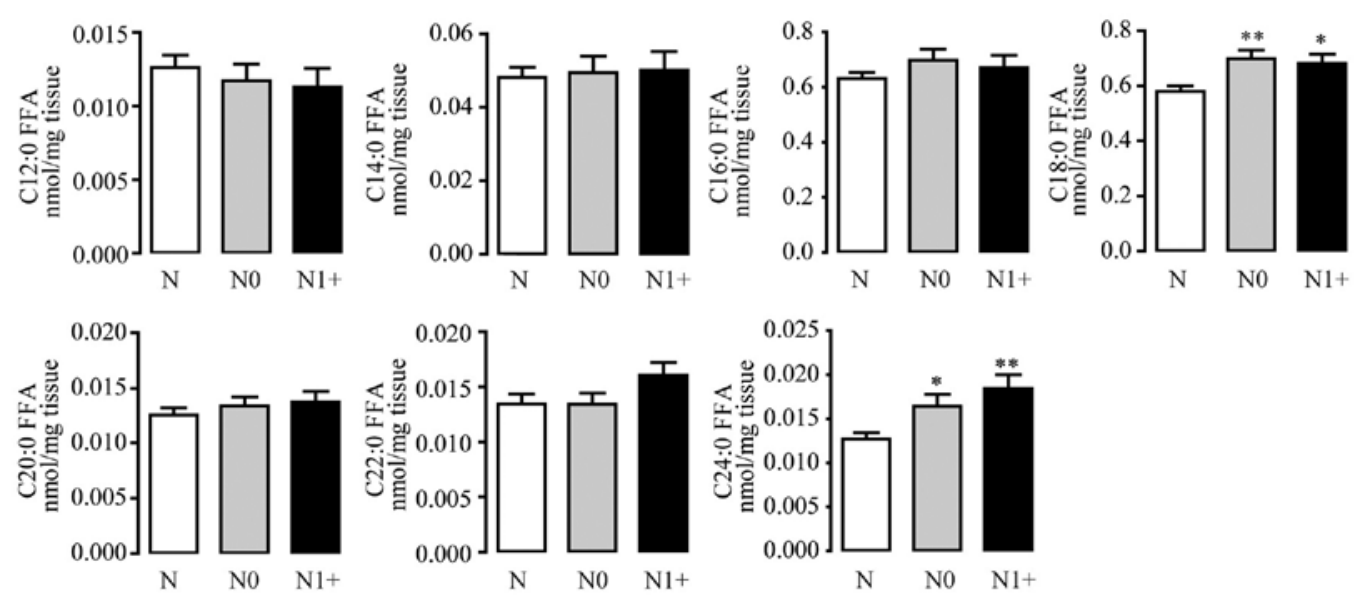

Figure 3. Levels of saturated fatty acids (SFAs) in human colon cancer tissues. No obvious variation of SFAs appeared, except for upregulated levels of C18:0 and C24:0 FFAs in CRC tissues without lymph node metastasis (N0, gray bar) and CRC with lymph node metastasis (N1+, solid bars), which were compared with the non-tumor colon tissue controls (N, open bars), ${ }^{*} \mathrm{p}<0.05,{ }^{* *} \mathrm{p}<0.01, \mathrm{n}=22-47$.
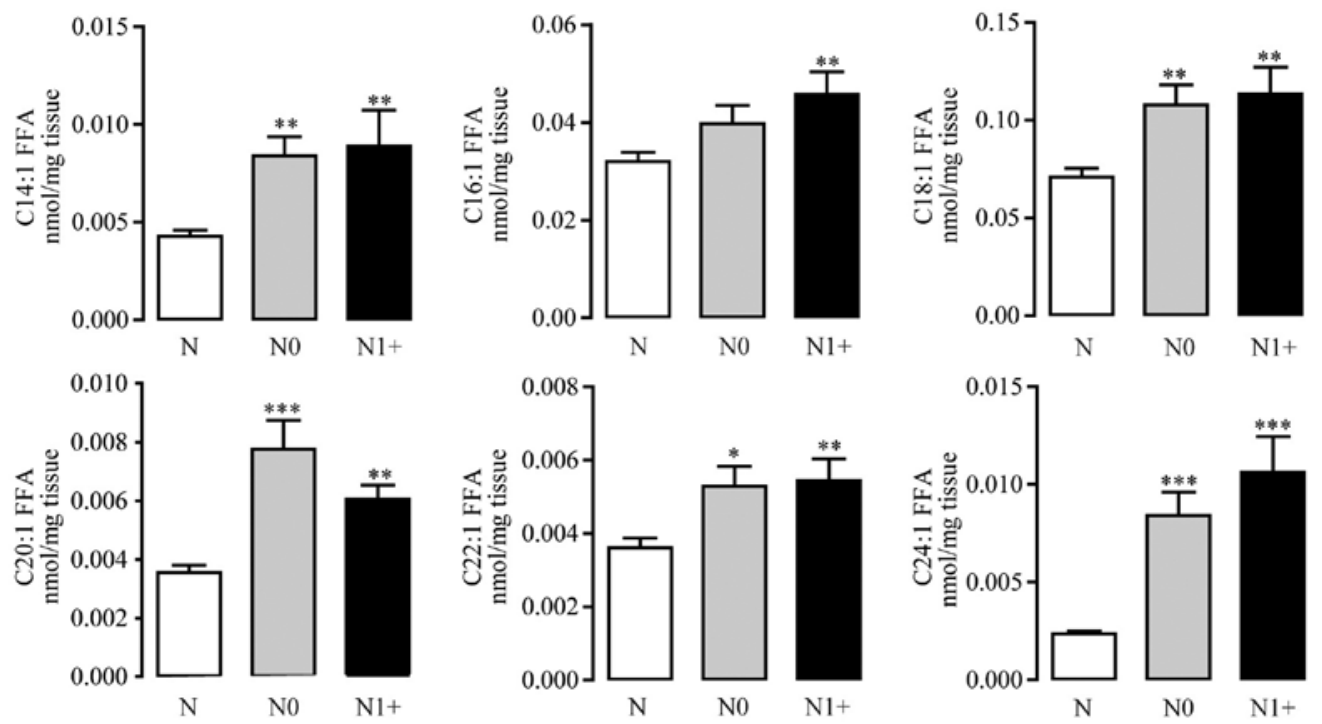

Figure 4. High expression levels of MUFAs in human colon cancer tissues. Levels of MUFAs with carbon chains from C14 to C24 were higher in the CRC tissues without lymph metastasis (N0, gray bar) and CRC tissues with lymph node metastasis (N1+, solid bars), which were compared with the non-tumor colon tissue controls (N, open bars), ${ }^{*} \mathrm{p}<0.05,{ }^{* *} \mathrm{p}<0.01,{ }^{* * *} \mathrm{p}<0.001, \mathrm{n}=22-47$. MUFAs, monounsaturated fatty acids.
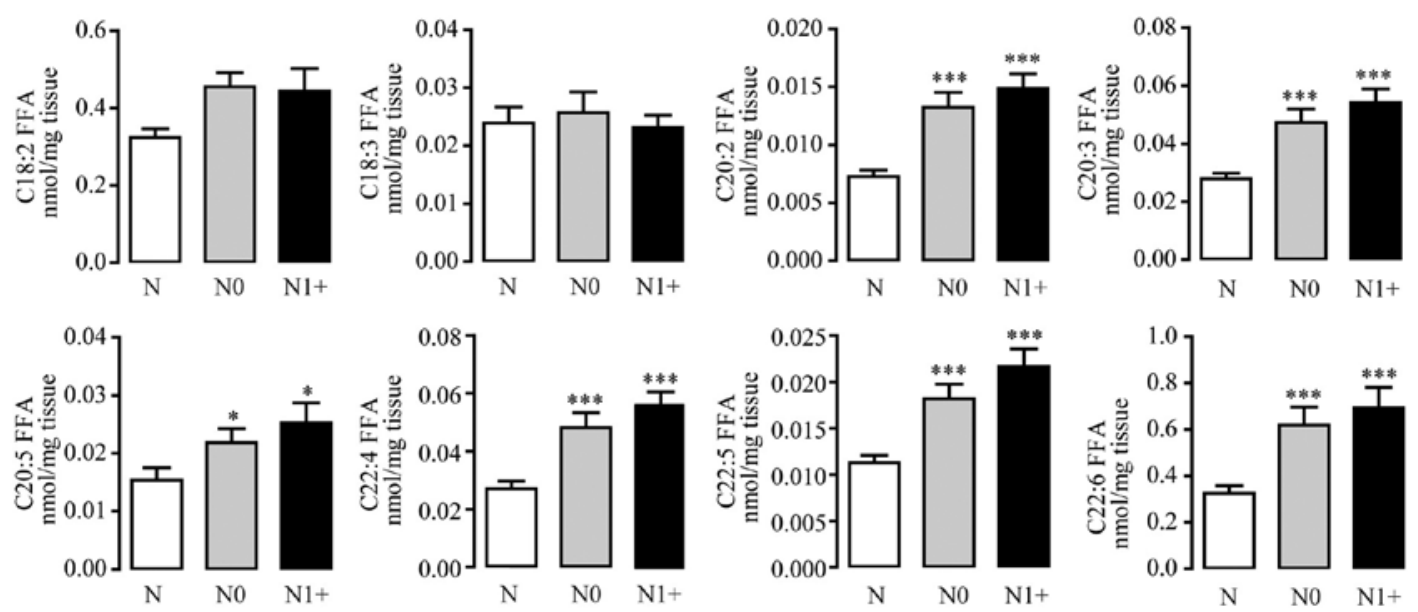

Figure 5. Levels of PUFAs in human colon cancer tissues. Except for C18 PUFAs, levels of most PUFAs with carbon chains from C14 to C24 were higher in the CRC tissues without lymph metastasis (N0, gray bar) and CRC tissues with lymph metastasis (N1+, solid bars), which were compared with the non-tumor colon tissue controls (N, open bars). ${ }^{*} \mathrm{p}<0.05,{ }^{* *} \mathrm{p}<0.01,{ }^{* * *} \mathrm{p}<0.001, \mathrm{n}=22-47$. PUFAs, polyunsaturated fatty acids. 
A
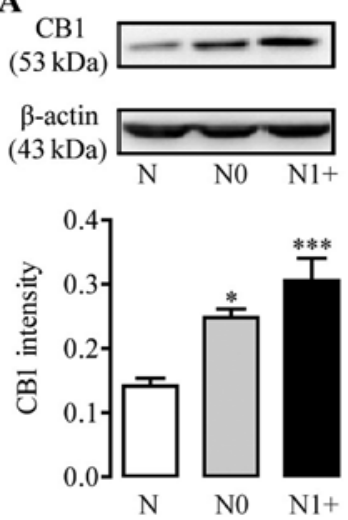

B

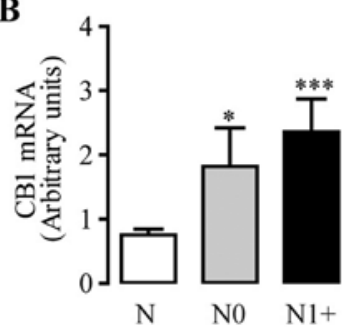

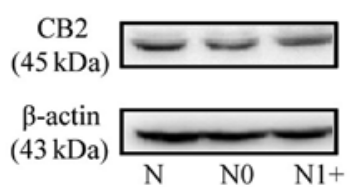
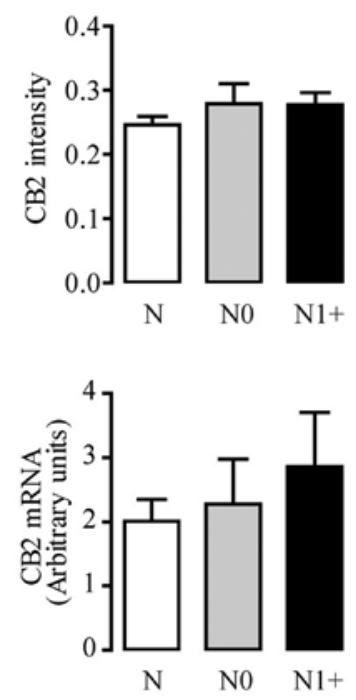

Figure 6. Expression levels of cannabinoid receptors in human colon cancer tissues. The protein expression levels (A) and mRNA expression levels (B) of $\mathrm{CB} 1$ and $\mathrm{CB} 2$ in the non-tumor colon tissue controls (N, open bars), $\mathrm{CRC}$ tissues without lymph metastasis (NO, gray bar) and CRC tissues with lymph node metastasis $\left(\mathrm{N} 1+\right.$, solid bars), ${ }^{*} \mathrm{p}<0.05,{ }^{* * *} \mathrm{p}<0.001, \mathrm{n}=22-47$.

Expression levels of cannabinoid receptors in human CRC tissues. Based on the alteration of endocannabinoid levels in the CRC tissues, we further analyzed the protein expression levels of the cannabinoid receptors via western blot analysis in the same tissue samples. CB1 expression was elevated 1.5 -fold in the N0 group $(0.25 \pm 0.01)$, and 2 -fold in the $\mathrm{N} 1+$ group $(0.31 \pm 0.04)$, which were compared with adjacent non-tumor samples $(0.14 \pm 0.01)$ (Fig. 6A). However, protein expression of CB2 showed no obvious alteration between the CRC tissues and non-tumor tissues (Fig. 6A). The mRNA levels of cannabinoid receptors were further measured. Consistent with the protein levels, the mRNA level of CB1 was also upregulated in the tumor samples, and the gene expression of the CB2 receptor was not altered (Fig. 6B). The detailed result of the CB1 mRNA levels was as follows: $0.8 \pm 0.1$ in the $\mathrm{N}$ group; $1.8 \pm 0.5$ in the N0 group; $2.4 \pm 0.5$ in the N1+ group.

\section{Discussion}

Endocannabinoids have been demonstrated to act with multi-function in the gastrointestinal tract, particularly in the colon. D'Argenio et al reported that in human colitis and inflammatory bowel diseases (IBDs), upregulation of AEA levels exerted a protective effect against the inflammatory response mainly via the CB1 receptor subtype (28). Since chronic inflammation is a recognized risk factor for CRC, the key role of the cannabinoid system in CRC progression warrants further investigation. It was verified that increased endocannabinoid levels reduced the development of precancerous lesions in the mouse colon (29). In addition, endocannabinoids were even found to inhibit the proliferation of cancer cells, such as breast

cancer, prostate cancer and colorectal cancer cells, mostly via CB1 receptors $(15,17,30)$.

In the present study, we found that the level of AEA was increased in the CRC tissues, particularly in those with lymph node metastasis, while 2-AG levels showed no significant difference. The result of AEA levels was consisting with data reported by Ligresti et al (15). In contast, 2-AG levels in their study were also upregulated, which may have been induced by differences in the sample source, population distribution and dietary habits. To further verify the detailed pathway of endocannabinoid alterations, we measured the expression of relevant metabolic enzymes. In agreement with the marked upward trends for AEA, the mRNA levels and activities of synthase NPLD were highly elevated. The same tendency was also observed for FAAH, which acts as an AEA hydrolase. In the present study, high expression of NPLD was thought to be the primary reason for elevation of AEA, thereby FAAH was enhanced as a positive feedback due to substrate accumulation. As a result, arachidonic acid, the main metabolite of AEA, was found to increase in the CRC tissues particularly in those associated with lymph node metastasis. Regarding the metabolic pathway of 2-AG, there was no significant change in the activities and the expression of synthase DGL- $\alpha$ and hydrolase MGL between the tumor and the control tissues, which may be a reasonable explanation for the consistent levels of 2-AG. Detailed mechanisms of the variation in endocannabinoid levels still require further study.

AEA elevation in CRC was suggested to be self-protection against further tumor progression. It is known that multiple pharmacological functions of AEA are accomplished through activation of cannabinoid receptors, particular CB1 $(12,13)$. In the present study, the mRNA level of CB1 was increased in the CRC tissues and even higher in the lymph node metastatic tumor tissues. Western blot analysis further supported the above results, which was similar to a research study by Cianchi et al (31). A report by Ligresti et al demonstrated that activation of the $\mathrm{CB} 1$ receptor resulted in inhibition of CRC cell proliferation in vitro (15), while the $\mathrm{CB} 2$ receptor did not display an obvious change in CRC cells, which is also consistent with our findings. Based on the present data, we suggest that strong activity of NPLD produced abundant endogenic AEA, which provided much more substrates to induce higher levels of FAAH, thereby sufficient agonist AEA further activated preferable $\mathrm{CB} 1$ receptor to prevent tumor progression.

It has been reported that $\mathrm{CB} 1$ receptor activation caused sphingomyelin hydrolysis and rapid ceramide production in astrocytes and C6 glioblastoma cells (20,32). Tumor apoptosis induced by cannabinoid receptor activation was verified to be mediated by ceramide de novo synthesis (31). Growing evidence suggests that as a ubiquitous lipid messenger, ceramides play an important role in the control of tumor cell fate (33). Yet there is limited research to further identify the role of ceramides in CRC progression and treatment. In the present study, we found that the levels of ceramides C16:0, C24:0 and C24:1 were obviously elevated in the CRC tissues. Increased levels of the $\mathrm{C} 16$ and $\mathrm{C} 24$ ceramides were also observed in human head and neck squamous cell carcinoma (HNSCC) (34). As we observed, the $\mathrm{C} 16$ ceramide is the most abundant ceramide in the colon, followed by the $\mathrm{C} 24$ ceramide. Both two types of ceramides were reported to induce apoptosis in hepatocellular 
cancer, prostate cancer and colon cancer cells (35-37). In another report, the $\mathrm{C} 16$ ceramide was also considered to play an anti-apoptotic role in HNSCC via selective regulation of the ER-stress response pathway (38). In contrary, levels of C18 and C20 ceramides in the CRC tumors were significantly decreased compared to these levels in the non-tumor tissues. A reduced level of $\mathrm{C} 18$ ceramide in tumors was also reported in HNSCC (34), which was thought to contend for tumor growth via telomerase activity and mitochondrial dysfunction.

We further measured ceramide synthases (CerSs) in human CRC tissues, which are important enzymes for ceramide synthesis. Since specificity of each CerS subtype for ceramides with fatty acyl chain, four abundant CerS subtypes in human colon were evaluated. Unexpectedly, the mRNA levels of all the detected CerSs were sharply increased in the CRC tissues. Upregulated levels of CerS2, CerS5 and CerS6 were explained to synthesize and elevate enough $\mathrm{C} 16$ and C24 ceramides to decelerate tumorigenesis. CerS1 showed a contrary upward trend against the reduced levels of its specific $\mathrm{C} 18$ ceramide, which may be a defensive mechanism to compensate enough $\mathrm{C} 18$ ceramides. Overlapping enzyme activity of CerS2 and CerS6, partly responsible for synthesis of $\mathrm{C} 18$ and $\mathrm{C} 20$ ceramides, made it difficult to study CerSs and ceramides in a one-to-one relationship. The complex regulatory mechanism of CerSs and ceramides with different carbon chains of fatty acyl still require further in-depth research. Yet, consistent alteration of C16/C18/C24 ceramides in several tumors revealed that they may be potential indicators in tumor monitoring.

In addition to AA, a metabolite of AEA, the expression of other free fatty acids was also detected by LC-MS/MS. MUFAs with carbon chains from $\mathrm{C} 14$ to $\mathrm{C} 24$ and most PUFAs showed a high unitive increase, while among the SFAs, only C18:0 and C24:0 FFAs were increased in the CRC tissues. Increased MUFAs provide enough resource to build more phospholipids, diacylglycerols and triacylglycerols, to serve as a continuous nutrient fuel for rapidly proliferating cancer cells $(39,40)$. As we know, regular cell growth needs a stabilized balance between SFAs and unsaturated fatty acids (UFAs) (41). The augmentation of MUFAs and PUFAs demonstrated the rapid proliferation and aggressiveness of human CRC.

In conclusion, the present study demonstrated an increase in AEA in human CRC tissues along with lymph node metastasis, which could be an endogenous bio-indicator for the malignant degrees of CRC. Overexpression of its preferred receptor, $\mathrm{CB} 1$, aided in further understanding the important role of the endocannabinoid system in the treatment of human CRC. Moreover, the variation in levels of long chain ceramides and UFAs revealed that lipid dysfunction plays a vital role in CRC progression, and these levels may also be utilized as important monitoring biomarkers and novel drug targets for colorectal cancer.

\section{Acknowledgements}

The authors thank the Eye Institute of Xiamen University for assistance and Mrs Jin Fu for help with the design of this research. This study was supported by grants from the National Natural Sciences Foundation of China (no. 81373273), Fujian Provincial Natural Science Foundation (no.
2013J05122), and Xiamen Southern Ocean Research Center Project (no. 14GYY018NF18).

\section{References}

1. Veldkamp R, Gholghesaei M, Bonjer HJ, Meijer DW, Buunen M, Jeekel J, Anderberg B, Cuesta MA, Cuschierl A, Fingerhut A, et al: European Association of Endoscopic Surgery (EAES): Laparoscopic resection of colon Cancer: Consensus of the European Association of Endoscopic Surgery (EAES). Surg Endosc 18: 1163-1185, 2004.

2. Tsujimoto H, Tsukioka S, Ono S, Sakamoto E, Sakamoto K, Tsuta K, Nakagawa F, Saito H, Uchida J, Kiniwa M, et al: Effect of leucovorin on the antitumor efficacy of the 5-FU prodrug, tegafur-uracil, in human colorectal cancer xenografts with various expression levels of thymidylate synthase. Oncol Lett 1: 973-980, 2010

3. Yang SY, Miah A, Sales KM, Fuller B, Seifalian AM and Winslet M: Inhibition of the p38 MAPK pathway sensitises human colon cancer cells to 5-fluorouracil treatment. Int J Oncol 38: 1695-1702, 2011.

4. Mechoulam R, Ben-Shabat S, Hanus L, Ligumsky M, Kaminski NE, Schatz AR, Gopher A, Almog S, Martin BR, Compton DR, et al: Identification of an endogenous 2-monoglyceride, present in canine gut, that binds to cannabinoid receptors. Biochem Pharmacol 50: 83-90, 1995.

5. Devane WA, Breuer A, Sheskin T, Järbe TU, Eisen MS and Mechoulam R: A novel probe for the cannabinoid receptor. J Med Chem 35: 2065-2069, 1992.

6. Di Carlo G and Izzo AA: Cannabinoids for gastrointestinal diseases: Potential therapeutic applications. Expert Opin Investig Drugs 12: 39-49, 2003.

7. De Petrocellis L, Cascio MG and Di Marzo V: The endocannabinoid system: A general view and latest additions. Br J Pharmacol 141: 765-774, 2004.

8. Piomelli D: The molecular logic of endocannabinoid signalling. Nat Rev Neurosci 4: 873-884, 2003.

9. Cravatt BF, Giang DK, Mayfield SP, Boger DL, Lerner RA and Gilula NB: Molecular characterization of an enzyme that degrades neuromodulatory fatty-acid amides. Nature 384: 83-87, 1996.

10. Pertwee RG (ed): Cannabinoid Receptors. Academic Press, New York, NY, 1995.

11. Pertwee RG: Pharmacology of cannabinoid CB1 and CB2 receptors. Pharmacol Ther 74: 129-180, 1997.

12. Di Marzo V, Melck D, De Petrocellis L and Bisogno T: Cannabimimetic fatty acid derivatives in cancer and inflammation. Prostaglandins Other Lipid Mediat 61: 43-61, 2000.

13. Gonsiorek W, Lunn C, Fan X, Narula S, Lundell D and Hipkin RW: Endocannabinoid 2-arachidonyl glycerol is a full agonist through human type 2 cannabinoid receptor: Antagonism by anandamide. Mol Pharmacol 57: 1045-1050, 2000.

14. Guzmán M: Cannabinoids: Potential anticancer agents. Nat Rev Cancer 3: 745-755, 2003.

15. Ligresti A, Bisogno T, Matias I, De Petrocellis L, Cascio MG, Cosenza V, D'argenio G, Scaglione G, Bifulco M, Sorrentini I, et al: Possible endocannabinoid control of colorectal cancer growth. Gastroenterology 125: 677-687, 2003.

16. Sarnataro D, Grimaldi C, Pisanti S, Gazzerro P, Laezza C, Zurzolo $\mathrm{C}$ and Bifulco M: Plasma membrane and lysosomal localization of CB1 cannabinoid receptor are dependent on lipid rafts and regulated by anandamide in human breast cancer cells. FEBS Lett 579: 6343-6349, 2005.

17. De Petrocellis L, Melck D, Palmisano A, Bisogno T, Laezza C, Bifulco $\mathrm{M}$ and Di Marzo V: The endogenous cannabinoid anandamide inhibits human breast cancer cell proliferation. Proc Natl Acad Sci USA 95: 8375-8380, 1998.

18. Contassot E, Wilmotte R, Tenan M, Belkouch MC, Schnüriger V, de Tribolet N, Burkhardt K and Dietrich PY: Arachidonylethanolamide induces apoptosis of human glioma cells through vanilloid receptor-1. J Neuropathol Exp Neurol 63: 956-963, 2004.

19. Galve-Roperh I, Sánchez C, Cortés ML, Gómez del Pulgar T, Izquierdo $\mathrm{M}$ and Guzmán M: Anti-tumoral action of cannabinoids: Involvement of sustained ceramide accumulation and extracellular signal-regulated kinase activation. Nat Med 6: 313-319, 2000. 
20. Morad SA and Cabot MC: Ceramide-orchestrated signalling in cancer cells. Nat Rev Cancer 13: 51-65, 2013.

21. Grösch S, Schiffmann S and Geisslinger G: Chain length-specific properties of ceramides. Prog Lipid Res 51: 50-62, 2012.

22. Selzner M, Bielawska A, Morse MA, Rüdiger HA, Sindram D, Hannun YA and Clavien PA: Induction of apoptotic cell death and prevention of tumor growth by ceramide analogues in metastatic human colon cancer. Cancer Res 61: 1233-1240, 2001.

23. Garzotto M, White-Jones M, Jiang Y, Ehleiter D, Liao WC, Haimovitz-Friedman A, Fuks Z and Kolesnick R: 12-O-tetradecanoylphorbol-13-acetate-induced apoptosis in LNCaP cells is mediated through ceramide synthase. Cancer Res 58: 2260-2264, 1998.

24. Chmura SJ, Nodzenski E, Beckett MA, Kufe DW, Quintans J and Weichselbaum RR: Loss of ceramide production confers resistance to radiation-induced apoptosis. Cancer Res 57: 1270-1275, 1997

25. Verheij M, van Blitterswijk WJ and Bartelink H: Radiationinduced apoptosis - the ceramide-SAPK signaling pathway and clinical aspects. Acta Oncol 37: 575-581, 1998

26. Chen L, Xie B, Li L, Jiang W, Zhang Y, Fu J, Guan G and Qiu Y: Rapid and sensitive LC-MS/MS analysis of fatty acids in clinical amples. Chromatographia 77: 1241-1247, 2014.

27. Wu X, Han L, Zhang X, Li L, Jiang C, Qiu Y, Huang R, Xie B, Lin Z, Ren J, et al: Alteration of endocannabinoid system in human gliomas. J Neurochem 120: 842-849, 2012.

28. D'Argenio G, Valenti M, Scaglione G, Cosenza V, Sorrentini I and Di Marzo V: Upregulation of anandamide levels as an endogenous mechanism and a pharmacological strategy to limit colon inflammation. FASEB J 20: 568-570, 2006.

29. Izzo AA, Aviello G, Petrosino S, Orlando P, Marsicano G, Lutz B, Borrelli F, Capasso R, Nigam S, Capasso F, et al: Endocannabinoid Research Group: Increased endocannabinoid levels reduce the development of precancerous lesions in the mouse colon. J Mol Med Berl 86: 89-98, 2008.

30. Melck D, De Petrocellis L, Orlando P, Bisogno T, Laezza C, Bifulco M and Di Marzo V: Suppression of nerve growth factor Trk receptors and prolactin receptors by endocannabinoids leads to inhibition of human breast and prostate cancer cell proliferation. Endocrinology 141: 118-126, 2000.

31. Cianchi F, Papucci L, Schiavone N, Lulli M, Magnelli L, Vinci MC, Messerini L, Manera C, Ronconi E, Romagnani P, et al: Cannabinoid receptor activation induces apoptosis through tumor necrosis factor $\alpha$-mediated ceramide de novo synthesis in colon cancer cells. Clin Cancer Res 14: 7691-7700, 2008
32. Sánchez C, Galve-Roperh I,Rueda D and Guzmán M: Involvement of sphingomyelin hydrolysis and the mitogen-activated protein kinase cascade in the $\Delta^{9}$-tetrahydrocannabinol-induced stimulation of glucose metabolism in primary astrocytes. Mol Pharmacol 54: 834-843, 1998.

33. Ogretmen B and Hannun YA: Biologically active sphingolipids in cancer pathogenesis and treatment. Nat Rev Cancer 4: 604-616, 2004.

34. Koybasi S, Senkal CE, Sundararaj K, Spassieva S, Bielawski J, Osta W, Day TA, Jiang JC, Jazwinski SM, Hannun YA, et al: Defects in cell growth regulation by $\mathrm{C} 18: 0$-ceramide and longevity assurance gene 1 in human head and neck squamous cell carcinomas. J Biol Chem 279: 44311-44319, 2004.

35. Seumois G, Fillet M, Gillet L, Faccinetto C, Desmet C, François C, Dewals B, Oury C, Vanderplasschen A, Lekeux P, et al: De novo C16- and C24-ceramide generation contributes to spontaneous neutrophil apoptosis. J Leukoc Biol 81: 1477-1486, 2007.

36. White-Gilbertson S, Mullen T, Senkal C, Lu P, Ogretmen B, Obeid L and Voelkel-Johnson C: Ceramide synthase 6 modulates TRAIL sensitivity and nuclear translocation of active caspase-3 in colon cancer cells. Oncogene 28: 1132-1141, 2009.

37. Osawa Y, Uchinami H, Bielawski J, Schwabe RF, Hannun YA and BrennerDA: Roles for C16-ceramide and sphingosine 1-phosphate in regulating hepatocyte apoptosis in response to tumor necrosis factor-alpha. J Biol Chem 280: 27879-27887, 2005.

38. Senkal CE, Ponnusamy S, Bielawski J, Hannun YA and Ogretmen B: Antiapoptotic roles of ceramide-synthase6-generated C16-ceramide via selective regulation of the ATF6/CHOP arm of ER-stress-response pathways. FASEB J 24: 296-308, 2010.

39. Cairns RA, Harris IS and Mak TW: Regulation of cancer cell metabolism. Nat Rev Cancer 11: 85-95, 2011.

40. Rysman E, Brusselmans K, Scheys K, Timmermans L, Derua R, Munck S, Van Veldhoven PP, Waltregny D, Daniëls VW, Machiels J, et al: De novo lipogenesis protects cancer cells from free radicals and chemotherapeutics by promoting membrane lipid saturation. Cancer Res 70: 8117-8126, 2010

41. Nashed M, Chisholm JW and Igal RA: Stearoyl-CoA desaturase activity modulates the activation of epidermal growth factor receptor in human lung cancer cells. Exp Biol Med 237: 1007$1017,2012$. 